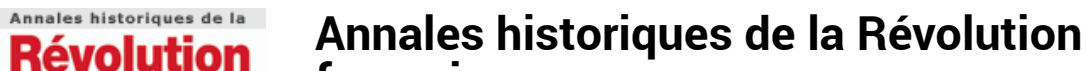 \\ française française
}

341 | juillet-septembre 2005

Varia

\section{Deux siècles de débats républicains (1792-2004)}

Jacques Bernet

\section{(2) OpenEdition}

\section{Journals}

Édition électronique

URL : https://journals.openedition.org/ahrf/2226

DOI : 10.4000/ahrf.2226

ISSN : 1952-403X

Éditeur :

Armand Colin, Société des études robespierristes

Édition imprimée

Date de publication : 1 septembre 2005

Pagination : 193-195

ISSN : 0003-4436

\section{Référence électronique}

Jacques Bernet, « Deux siècles de débats républicains (1792-2004)», Annales historiques de la

Révolution française [En ligne], 341 | juillet-septembre 2005, mis en ligne le 27 avril 2006, consulté le 23 avril 2022. URL : http://journals.openedition.org/ahrf/2226 ; DOI : https://doi.org/10.4000/ahrf.2226

Ce document a été généré automatiquement le 23 avril 2022.

Tous droits réservés 


\title{
Deux siècles de débats républicains (1792-2004)
}

\author{
Jacques Bernet
}

\section{RÉFÉRENCE}

Paul Baquiast, dir., Deux siècles de débats républicains (1792-2004), Paris, L'Harmattan, 2004, 197 p., ISBN 2-7475-6664-1, 17.50 e.

Ce petit ouvrage rassemble les communications d'un séminaire organisé par l'Association des amis d'Eugène et Camille Pelletan (AECP) sur le vaste thème de la République en France. Eugène Pelletan (1813-1884) et son fils Camille (1846-1914) ont été parmi les pères fondateurs du radicalisme républicain sous le second Empire et la IIIe République : le premier, journaliste et auteur de La Profession de foi du 19e siècle, théoricien du «Progrès continu», fut ministre de l'Instruction Publique dans le gouvernement de Défense nationale de Gambetta en 1870-1871; le second aussi journaliste, député radical de 1881 à 1912, appartint au ministère anti-clérical d'E. Combes, où il fut un ministre de la Marine contesté à l'époque de l'affaire des fiches. Comme le précise P. Baquiast dans son introduction, ce recueil de communications se veut « un livre politique au service de l'idée républicaine ; livre citoyen au service de la compréhension des débats du forum; livre universitaire au service de la connaissance ». Il y a là un mélange des genres qui peut évidemment présenter des risques déontologiques, d'autant que plusieurs des auteurs, appartenant pour une part à la communauté universitaire française ou étrangère, ont des engagements politiques, plus particulièrement dans la mouvance radicale valoisienne - le plus connu étant le criminologue Alain Bauer, ancien Grand Maitre du Grand Orient de France, devenu président de l'Observatoire de la Délinquance. Mais les étiquettes importent moins que la validité du contenu historique, toujours susceptible d'une lecture «scientifique » et critique, sinon "objective», sur des sujets restant tout aussi passionnés que le jacobinisme, la laïcité, l'école, la décolonisation, la citoyenneté des femmes, la construction européenne ou la mondialisation, et plus largement sur le contenu même 
du pacte républicain, par delà le consensus apparent et quelque peu trompeur dont il bénéficie de nos jours dans l'opinion. La démarche régressive généralement adoptée par les auteurs, qui fut d'ailleurs celle de Marc Bloch dans ses « caractères originaux de l'agriculture française ", correspond au questionnement légitime des contemporains, tant politiques qu'historiens, mais invite à d'autant plus de prudence qu'elle accroît le danger de relecture anachronique du passé, un écueil auquel tous n'ont pas su toujours échapper.

Sudhir Hazarresingh, professeur de sciences politiques au Balliol College à Oxford, suit jusqu'à nos jours les avatars d'un jacobinisme pluriel en France, qu'il définit comme une «sensibilité » plutôt qu'une idéologie, dans ses continuités, diversité et changements, distinguant trois tendances complémentaires et concurrentes"républicaine modérée ", "révolutionnaire » et "napoléonienne »- dont il souligne aussi les souplesses et les possibles convergences : «Le Jacobinisme d'État [...] ce sont des républicains modérés utilisant le langage et la symbolique révolutionnaire pour faire une politique bonapartiste» (p. 22). L'auteur montre que les Jacobins au pouvoir ont su se montrer plus pragmatiques qu'idéologues et défend de manière convaincante le paradoxe des "effets bénéfiques» des "caractéristiques négatives" (rigidité, élitisme, intolérance) du Jacobinisme pour "construire une nation » et "contribuer positivement à la culture politique française »: «sans le jacobinisme, la France ne serait pas ce qu'elle est ; mais c'est aussi parce qu'elle est ce qu'elle est - querelleuse, divisée, fragmentée - que la France a pu puiser dans ses jacobinismes et y trouver les recours nécessaires pour suppléer à ses faiblesses sociales et culturelles». Tout en soulignant sa pesanteur dans les mentalités et la culture politique hexagonale, comme l'a encore illustré l'affaire du voile islamique, l'auteur souligne à raison l'épuisement du courant, tourné vers un passé mythifié devenu un frein à la nécessaire rénovation politique française. À cette réflexion globalement stimulante, succèdent les propos plus convenus de Serge Toursel sur la laïcité de 1789 à 2004, où la (faible) argumentation historique cède le pas à un discours idéologique d'intransigeance laïque défensive, sinon paranoïaque, récusant la notion de laïcité « ouverte ", comme s'il ne s'agissait de l'essence même de la (bienveillante) neutralité ou plutôt impartialité de l'État à l'égard de la liberté des croyances et des cultes.

3 Paul Baquiast reprend l'histoire de «l'École de la République » de la Constitution de 1791 à nos jours, entre "méritocratie, démocratisation, massification ", à partir des querelles que suscite sa crise actuelle dans le camp républicain lui-même, entre tenants des «savoirs" et de la "pédagogie ». Remontant aux sources des projets scolaires révolutionnaires, appliqués très progressivement au xix siècle, il démonte les mythes d'une école Jules Ferry "démocratique et méritocratique », fondée en réalité sur une forte ségrégation sociale, que l'on s'est s'efforcé de réduire au $\mathrm{xx}^{\mathrm{e}}$ siècle, par l'unification de l'École et la création du collège unique en 1975, non sans désillusions, quant aux effets d'une "démocratisation" confondue avec «massification" et prétendant imposer à tous un enseignement resté élitiste. Il en conclut non sans bon sens que « le débat entre savants et pédagogues est inscrit à l'intérieur même de l'esprit de chacun des acteurs du système éducatif [...] déchirés entre deux cultures: l'une tournée vers un passé largement mythifié, l'autre vers un avenir de réformes lourdes de désillusions », opposant deux conceptions de l'École de la République : «Pour les savants, elle s'inscrit dans un monde abstrait peuplé d'élèves idéaux dont il suffirait de décréter l'égalité pour qu'elle s'établisse. Pour les pédagogues, elle s'inscrit dans le réel, 
un réel qu'il s'agit de prendre en compte pour mieux le transformer et atteindre l'idéal ».

4 Passons sur les quelques pages plutôt confuses du constitutionnaliste Stéphane Baumont, opposant République et démocratie et sacralisant à l'excès la Constitution de 1958, pour aborder, avec Diane Le Béguec, la dialectique classique entre «ordre et liberté ", qu'elle traite sous l'angle des principes plus que de manière historique. Alain Bauer qui suit les avatars de la police de 1789 à nos jours, comme «institution (relativement) républicaine en débat ", rappelle ses racines municipales sous l'Ancien Régime et la Révolution, avec la transformation des milices bourgeoises en Gardes nationales, la création d'une véritable police d'État sous le régime de Vichy renforçant l'aspect politique de sa vocation d'ordre public, d'où la réapparition de polices municipales à partir des années 1980, en réponse à une poussée de la criminalité, de la délinquance et de la violence urbaine, dont l'auteur présente un tableau quelque peu noirci, qui mériterait sans doute débat, quant à leurs mesure, causes et remèdes. La communication de Bruno Marnot «Les républicains devant la science et la technique : l'exemple des chemins de fer, 1830-1937) » est, à notre sens, une des plus originales et stimulantes du recueil, suggérant à propos de cette réalisation majeure de la Révolution industrielle, l'essence des débats des républicains quant au bon usage du progrès, entre "partisans de la nécessité économique et de la justice sociale», tenants de «l'universalité du progrès technique » ou de la «responsabilité de l'État » garant des services publics.

5 Abordant les positions des Républicains hexagonaux sur "la question de la femme » entre 1852 et 1914, Judith $\mathrm{F}$. Stone n'a guère de mal à démontrer « la force du sexisme de l'idéologie républicaine » en France, en dépit de Condorcet ou F. Buisson, traduite par le refus persistant d'accorder la pleine citoyenneté à la seconde moitié du genre humain, ce qui constitue sans doute la plus authentique et bien peu reluisante " exception française ", comme a pu le souligner Jean Baubérot. Les non moins grandes ambiguïtés des républicains français face " au fait colonial et à la décolonisation ", de 1792 à 1962, sont aussi suggérées par l'étude malheureusement trop brève et fragmentaire de Paul Marcus, car la IIIe République démocratique et égalitaire en métropole s'intitulait Empire dans ses possessions extérieures, où elle maintint jusqu'au bout l'inégalité de statut et de condition sociale. Galina Kaninskaya, historienne du courant radical en France après 1945, remémore les divisions de ce parti à propos de la C.E.D. dans les années 1950-1954, ayant contribué à un échec du traité, au détriment de la construction européenne. Enfin l'intéressante étude de Stéphane Lucas sur l'attitude du camp républicain face à la "première mondialisation (1870-1914)", nous rappelle que nos défis actuels ne sont pas dépourvus de précédents, dont on peut tirer des leçons[䂑 : "La façon dont les républicains ont su concilier progrès social et mondialisation devrait inspirer ceux qui aujourd'hui cherchent des réponses à la deuxième vague de mondialisation des échanges que nous connaissons » (p. 179).

Dans sa diversité et son hétérogénéité, après $\mathrm{C}$. Nicolet, $\mathrm{V}$. Duclert et $\mathrm{C}$. Prochasson, ce volume apporte sa pierre aux débats sur l'essence, les combats et les débats du républicanisme français, nous renseignant d'ailleurs au moins autant sur leur perception actuelle dans une frange de l'intelligentsia se réclamant de sa longue tradition, que sur ses aspects proprement historiques, sur lesquels il reste bien des choses à défricher et approfondir. 